\title{
Schwinger effect in compact space: A real time calculation
}

\author{
Yue Qiu $\odot^{*}$ and Lorenzo Sorbo ${ }^{\dagger}$ \\ Amherst Center for Fundamental Interactions, Department of Physics, \\ University of Massachusetts, Amherst, Massachusetts 01003, U.S.A
}

(Received 2 June 2020; accepted 22 July 2020; published 10 August 2020)

\begin{abstract}
We compute the discharging rate of a uniform electric field due to Schwinger pair production in $(1+1)$ dimensional scalar electrodynamics with a compact dimension of radius $R$. Our calculation is performed in real time, using the in-in formalism. For large compactification radii, $R \rightarrow \infty$, we recover the standard noncompact space result. However, other ranges of values of $R$ and of the mass $m$ of the charged scalar give rise to a richer set of behaviors. For $R \gtrsim \mathcal{O}(1 / m)$ with $m$ large enough, the electric field oscillates in time, whereas for $R \rightarrow 0$ it decreases in steps. We discuss the origin of these results.
\end{abstract}

DOI: 10.1103/PhysRevD.102.045010

\section{INTRODUCTION}

The possibility of creating matter in the presence of a strong external field is a remarkable feature of relativistic quantum fields. The earliest and possibly most studied example of such phenomenon is the Schwinger effect [1,2]. In this process, an external electric field accelerates charged virtual particles, extracting them from the vacuum and turning them into real pairs. The pair of produced particles generates an electric field that opposes the field responsible for their creation, reducing its intensity. The energy in the electric field lost this way is thus converted into the energy in the particles.

The rate of pair production can be computed using different techniques, such as the early tunneling calculation of Sauter [1], Schwinger's proper time method [2], instantons [3] and real time techniques [4-9]. The Schwinger effect in compact spaces has received significant attention only in more recent years. In Ref. [10] the system was studied using the instanton formalism, and it was argued that for small compactification radii the expression of the rate of pair production changes significantly from the one found in the noncompact case. The work [11] discussed the effect from the point of view of the effective lowerdimensional theory. The paper [12] studied numerically the unwinding of the electric flux as the produced pair circles multiple times the compact space. References [13,14] discuss Schwinger pair production on compact spaces with two compact dimensions. The Schwinger effect at finite

\footnotetext{
yqiu@umass.edu

†sorbo@physics.umass.edu
}

Published by the American Physical Society under the terms of the Creative Commons Attribution 4.0 International license. Further distribution of this work must maintain attribution to the author(s) and the published article's title, journal citation, and DOI. Funded by SCOAP. temperature has received more attention $[10,11,15-19]$ and is related to the spatially compact case as finite temperature effects can be captured by compactifying Euclidean time.

In the present paper we study the Schwinger effect in compact space by directly computing, for the first time, the number of produced pairs as a function of time. More specifically, we compute the change, to leading order, in the intensity of the electric field induced by the current of produced pairs. We perform our calculation using the in-in formalism [20,21], that at leading order corresponds to the calculation of Bogolyubov coefficients. As a consequence, our calculation parallels the one performed, for the noncompact case, in [4-9]. The system considered throughout the paper is scalar electrodynamics in $1+1$ spacetime dimensions, where the scalars have mass $m$ and charge $e$, and we denote by $E$ the magnitude of the background field.

Compactification of the spatial dimension implies that we can write the correction to the electric field as a series of contributions from the Kaluza-Klein modes of the charged field. The series is divergent, a consequence of the assumption that the background field is constant, so it had an infinite time to produce pairs. We isolate the divergent part, leaving the physical effect in the form of a convergent series, that can be in general evaluated numerically. Besides the renormalization associated to the infinite duration of the process, evaluation of the rate of pair production requires a different, more subtle kind of subtraction. Already in the noncompact case, a direct computation of the rate of pair production in the in-in formalism gives a nonvanishing result even in the decoupling limit where the mass $m$ of the produced particles diverges. This unphysical behavior is taken care of by using the formalism of the Bogolyubov coefficients, where twopoint functions are computed in terms of the normalordered ladder operators defined in the far future. Such an operation is equivalent to subtracting, from the twopoint functions computed in terms of the exact mode 
functions, the same two-point functions written in terms of the mode functions evaluated in the adiabatic approximation $[22,23]$.

While in the noncompact case the electric field discharges at a uniform rate, the evolution of the electric field has a less trivial behavior in the small radius regime $R \rightarrow 0$. As one can see in Fig. 2, the field is discharged by the effect of pair production in a stepwise fashion. While this is inconsistent with the assumption of a time-translation invariant background that would call for a uniform rate, we will argue in Sec. VA that this behavior is not unusual for a process of particle creation.

Another interesting behavior is that found in the regime $e E R^{2}=\mathcal{O}(1), m R \gtrsim \mathcal{O}(1)$. For this choice of parameters, the electric field performs oscillations that, even if their amplitude is exponentially small, are parametrically larger than its decrease due to pair production-see Fig. 3. As we discuss in Sec. V B, this behavior confirms the analysis of [11]. In particular, the oscillations are a direct consequence of the shape of the effective potential of the electric field obtained in the dimensionally reduced theory, after integrating out the modes of the charged field. In other words, in this regime the effect on the evolution of the electric field is dominated by the effects of virtual pairs of scalar quanta, and not by the creation of actual pairs of particles. Our results thus confirm the interpretation [11] of the statement of [10] that, for small radii, the electric field evolves at a rate whose expression is different from the one found in the noncompact theory. More specifically, while the expression of the net rate of pair production is unchanged, the amplitude of the oscillations of the electric field is larger and matches the amplitude of the effect discussed in [10].

The plan of the paper is as follows. In Sec. II we set up our formalism and reobtain, using the in-in formalism, well-known results on the rate of Schwinger pair production in noncompact space. In Sec. III we present new formulas for the rate of pair production in real time in compact space. Details of the calculation are presented in two Appendixes. In Sec. IV we analyze, in some representative regimes, the behavior of the formulas found in the previous section. We discuss our results in Sec. V.

\section{NONCOMPACT CASE}

Before investigating case of a compact spatial dimension, here we review the real-time analysis of the Schwinger effect in $1+1$ noncompact dimensions. Our Lagrangian for scalar electrodynamics reads

$$
\begin{aligned}
\mathcal{L}= & -\frac{1}{4} F_{\mu \nu} F^{\mu \nu}-\left(\partial_{\mu}-i e A_{\mu}\right) \phi\left(\partial^{\mu}+i e A^{\mu}\right) \phi^{*}-m^{2}|\phi|^{2} \\
= & \frac{1}{2} \dot{A}^{2}+|\dot{\phi}|^{2}-\left|\phi^{\prime}\right|^{2}-m^{2}|\phi|^{2} \\
& +i e A\left(\phi \phi^{\prime *}-\phi^{\prime} \phi^{*}\right)-e^{2} A^{2}|\phi|^{2}
\end{aligned}
$$

where we have chosen the gauge $A_{\mu}=(0, A)$ [12] and where a overdot and a prime denote, respectively, a time and a space derivative. We then decompose the gauge field $A$ into a background yielding a constant electric field $E$ and a perturbation,

$$
A(x, t)=-E t+\delta A(x, t),
$$

so that the Lagrangian (1) takes the form,

$$
\begin{aligned}
\mathcal{L} \equiv & \mathcal{L}_{F}+\mathcal{L}_{I}=\frac{1}{2} \delta \dot{A}^{2}+|\dot{\phi}|^{2}-\left|\phi^{\prime}\right|^{2}-m^{2}|\phi|^{2} \\
& -i e E t\left(\phi \phi^{\prime *}-\phi^{\prime} \phi^{*}\right)-e^{2} E^{2} t^{2}|\phi|^{2} \\
& +i e \delta A\left(\phi \phi^{\prime *}-\phi^{\prime} \phi^{*}\right)-e^{2} \delta A^{2}|\phi|^{2}+2 e^{2} \delta A E t|\phi|^{2},
\end{aligned}
$$

where $\mathcal{L}_{F}$ is the free part of the Lagrangian, given in the first line of Eq. (3), whereas $\mathcal{L}_{I}$ denotes the interaction terms, given in the second line.

\section{A. The correction to the electric field in the in-in formalism}

We now quantize the fields $\phi$ and $\delta A$ and compute the correction to the electric field to leading order in the in-in formalism. As we will show in Sec. IID below, the resulting correction to the electric field accounts for the creation of pairs of charged scalars through the Schwinger mechanism.

The free equation for $\delta A$ is just $\delta \ddot{A}=0$, that has the general solution $\delta A(x, t)=\hat{c}(x)+\hat{d}(x) t$, where $\hat{c}(x)$ and $\hat{d}(x)$ are operators. Canonical quantization requires

$$
\begin{aligned}
{[\hat{c}(x)+\hat{d}(x) t, \hat{d}(y)] } & =i \delta(x-y), \\
{[\hat{c}(x)+\hat{d}(x) t, \hat{c}(y)+\hat{d}(y) t] } & =[\hat{d}(x), \hat{d}(y)]=0,
\end{aligned}
$$

that is satisfied by imposing the commutation relations,

$[\hat{c}(x), \hat{d}(y)]=i \delta(x-y),[\hat{c}(x), \hat{c}(y)]=[\hat{d}(x), \hat{d}(y)]=0$.

Next, we decompose the field $\phi$ as

$$
\begin{aligned}
\phi(x, t) & \equiv \int \frac{d k}{\sqrt{2 \pi}} e^{i k x} \hat{\phi}(k, t) \\
& =\int \frac{d k}{\sqrt{2 \pi}} e^{i k x}\left(\phi(k, t) \hat{a}_{k}+\phi(-k, t)^{*} \hat{b}_{-k}^{\dagger}\right),
\end{aligned}
$$

where the mode function $\phi(k, t)$ solves the equation of motion, derived from the free Lagrangian $\mathcal{L}_{F}$,

$$
\ddot{\phi}(k, t)+\left[(k+e E t)^{2}+m^{2}\right] \phi(k, t)=0 .
$$


The general solution of this equation can be written in terms of parabolic cylinder functions,

$$
\begin{aligned}
\phi(k, t)= & C_{1} D_{i \frac{m^{2}}{2 e E}-\frac{1}{2}}\left((k+e E t) \sqrt{\frac{2}{e E}} e^{-i \pi / 4}\right) \\
& +C_{2} D_{i \frac{m^{2}}{2 e E}-\frac{1}{2}}\left(-(k+e E t) \sqrt{\frac{2}{e E}} e^{-i \pi / 4}\right),
\end{aligned}
$$

where the integration constants $C_{1,2}$ can be fixed, after imposing the commutation relations $\left[\hat{a}_{k}, \hat{a}_{k^{\prime}}^{\dagger}\right]=\left[\hat{b}_{k}, \hat{b}_{k^{\prime}}^{\dagger}\right]=$ $\delta\left(k-k^{\prime}\right)$, by requiring positive frequency modes at early times, when the WKB approximation can be applied

$$
\begin{aligned}
\phi(k, t \rightarrow-\infty) & \rightarrow \phi^{\mathrm{ad}}(k, t), \quad \text { where } \\
\phi^{\mathrm{ad}}(k, t) & \equiv \frac{1}{\sqrt{2 \omega}} e^{-i \int \omega d t} \\
& \approx \frac{1}{\sqrt{2|e E t|}} e^{i \frac{1}{2 e E}(k+e E t)^{2}}|k+e E t|^{i \frac{m^{2}}{2 e E}}
\end{aligned}
$$

is the solution of the mode equations in the WKB approximation. This gives $\phi(k, t)=\frac{e^{-\frac{\pi m^{2}}{8 e E}}}{(2 e E)^{1 / 4}} D_{i \frac{m^{2}}{2 e E}-\frac{1}{2}}\left(-(k+e E t) \sqrt{\frac{2}{e E}} e^{-i \pi / 4}\right)$.

We compute the correction to electric field using the in-in formalism [20,21], in which the expectation value of an operator $\mathcal{O}(t)$ is given by

$$
\begin{aligned}
\langle\mathcal{O}(t)\rangle= & \sum_{N}(-i)^{N} \int^{t} d t_{1} \ldots \\
& \times \int^{t_{N-1}} d t_{N}\left\langle\left[\left[\ldots\left[\mathcal{O}_{\text {free }}(t), H_{\text {int }}\left(t_{1}\right)\right], \ldots\right], H_{\text {int }}\left(t_{N}\right)\right]\right\rangle,
\end{aligned}
$$

where $H_{\text {int }}(t)$ denotes the interaction Hamiltonian, and $\mathcal{O}_{\text {free }}$ represents the operator $\mathcal{O}$ computed in terms of the free mode functions.

In our case, the operator we are interested in is the correction to the electric field, $\delta E(x, t) \equiv-\delta \dot{A}(x, t)$. To lowest order in the in-in expansion, we only need to consider the cubic part of the interaction Hamiltonian,

$$
\begin{aligned}
H_{\mathrm{int}}^{(3)}= & -e \int d y \int \frac{d p d q}{(2 \pi)} e^{i(p-q) y} \\
& \times[(p+q)+2 e E t] \delta A(y) \hat{\phi}(p) \hat{\phi}^{\dagger}(q) .
\end{aligned}
$$

Hence the first order correction to the electric field reads

$$
\begin{aligned}
\langle\delta E(x, t)\rangle_{(1)} & =-i e \int^{t} d t_{1} \int d y \int \frac{d p d q}{(2 \pi)} e^{i(p-q) y}\left\langle\left[\delta \dot{A}(x, t), \delta A\left(y, t_{1}\right) \hat{\phi}\left(p, t_{1}\right) \hat{\phi}^{\dagger}\left(q, t_{1}\right)\right]\right\rangle\left((p+q)+2 e E t_{1}\right) \\
& =-e \int^{t} d t_{1} \int \frac{d p d q}{(2 \pi)} e^{i(p-q) x}\left\langle\hat{\phi}\left(p, t_{1}\right) \hat{\phi}^{\dagger}\left(q, t_{1}\right)\right\rangle\left[(p+q)+2 e E t_{1}\right],
\end{aligned}
$$

that could have also been obtained by integrating Gauss' law.

\section{B. The two-point function of the scalar. Vacuum subtraction}

We must now compute the two point function $\left\langle\hat{\phi}(p, t) \hat{\phi}^{\dagger}(q, t)\right\rangle$. Since this quantity does not vanish even when the system is in its vacuum, we need to subtract its vacuum component. We perform this subtraction by using the standard method of the Bogolyubov coefficients (see [5] for a nice discussion in this context).

The idea beyond the use of the Bogolyubov coefficients is that the concept of particles is well defined only when $\omega$ is evolving adiabatically. An especially heuristic argument for this invokes Heisenberg's uncertainty principle. The number of nonrelativistic particles in a box can be determined by measuring their total mass (with an uncertainly smaller than the mass $m$ of one particle) and dividing by the mass of one particle. The uncertainty principle tells us that the time $\Delta T$ needed to perform this measurement must be larger than $\hbar /\left(m c^{2}\right)$. Now, if the effective mass of the particles is time-dependent, then $\Delta T$ must be smaller than the time scale over which $m$ evolves, $\Delta T \lesssim m / \dot{m}$. We thus obtain the condition $\dot{m} / m^{2} \lesssim c^{2} / \hbar$, or, generalizing to the relativistic regime and going back to natural units, $\dot{\omega} \lesssim \omega^{2}$. In the adiabatic regime the general solution of the equations of motion for the mode functions is given by the linear combination $\quad \alpha(k) \phi^{\text {ad }}(k, t)+\beta(-k) \phi^{\text {ad }}(-k, t)^{*}, \quad$ where $\phi^{\text {ad }}(k, t)$ is defined in Eq. (9) and where $\alpha(k)$ and $\beta(k)$, known as Bogolyubov coefficients, are arbitrary constants. This motivates the following decomposition:

$$
\phi(k, t)=\alpha(k, t) \phi^{\mathrm{ad}}(k, t)+\beta(-k, t) \phi^{\mathrm{ad}}(-k, t)^{*},
$$


where $|\alpha(k, t)|^{2}-|\beta(k, t)|^{2}=1$ to maintain the correct normalization of the creation/annihilation operators $\left[\hat{a}_{k}, \hat{a}_{q}^{\dagger}\right]=$ $\delta(k-q)$ etc. Note that in (14) the quantities $\alpha(k, t)$ and $\beta(k, t)$ are assumed to depend also on time, so that this expression of $\phi(k, t)$ can be valid also when $|\dot{\omega}| \gtrsim \omega^{2}$. It is worth stressing here that we are assuming that $\phi^{\text {ad }}(k, t)$ is evaluated to leading order in the adiabatic approximation, but that in general higher order expressions might be more appropriate [24-28].

As long as the evolution of the mode functions is adiabatic, the choice of the parameters $\alpha(k)$ and $\beta(k)$ is arbitrary and irrelevant-provided, of course, also the Hilbert space of the system is defined accordingly (in particular, it is important that the system is evolving adiabatically at early times, $t \rightarrow-\infty$, so that we can define an initial vacuum state). The usual choice is $\alpha(k)=1$ and $\beta(k)=0$ as $t \rightarrow-\infty$, so that the vacuum is annihilated by $\hat{a}_{k}$. Now, if the system goes through a stage of nonadiabatic evolution, $\dot{\omega} \gtrsim \omega^{2}$, followed by a second and final period of adiabatic evolution, then in this second period $t \rightarrow+\infty$ the mode functions will have the form (14) with $\alpha(k, t)$ and $\beta(k, t)$ constant. Since we have already fixed $\alpha(k, t \rightarrow-\infty) \rightarrow 1$ and $\beta(k, t \rightarrow-\infty) \rightarrow 0$, however, we do not have the freedom to redefine $\alpha(k, t \rightarrow+\infty)$ and $\beta(k, t \rightarrow+\infty)$.

Let us now consider a set of observers born after the period of nonadiabaticity, during the $t \rightarrow+\infty$ epoch. These observers will see the mode functions of the form $\phi^{\text {ad }}(k, t)$ and will decompose the field as $\hat{\phi}(k, t)=\phi^{\text {ad }}(k, t) \hat{\circledast}_{k}+$ $\phi^{\text {ad }}(-k, t)^{*} \hat{\mathfrak{b}}_{-k}^{\dagger}$, where $\hat{\mathbb{a}}_{k}$ and $\hat{\mathbb{b}}_{k}$ are the annihilation operators for those observers, who will not even know that at early times, before they were born, the annihilation operators were $\hat{a}_{k}$ and $\hat{b}_{k}$. To make the definition of $\hat{a}_{k}$ and $\hat{\mathbb{b}}_{k}$ valid even at intermediate times, we define more in general,

$$
\hat{\phi}(k, t)=\phi^{\mathrm{ad}}(k, t) \hat{\mathrm{a}}_{k}(t)+\phi^{\mathrm{ad}}(-k, t)^{*} \hat{\mathrm{b}}_{-k}(t)^{\dagger} .
$$

This is equivalent to redefining

$$
\begin{aligned}
& \hat{\mathbb{a}}_{k}(t)=\alpha(k, t) \hat{a}_{k}+\beta(k, t)^{*} \hat{b}_{-k}^{\dagger} \\
& \hat{\mathbb{b}}_{k}(t)=\beta(k, t)^{*} \hat{a}_{-k}^{\dagger}+\alpha(k, t) \hat{b}_{k} .
\end{aligned}
$$

In particular, observers born at $t \rightarrow+\infty$ will normal order the operators $\hat{\mathbb{a}}_{k}$ and $\hat{\mathbb{b}}_{k}$, and not $\hat{a}_{k}$ and $\hat{b}_{k}$. These considerations motivate us to compute the quantity $\left\langle\hat{\phi}\left(p, t_{1}\right) \hat{\phi}^{\dagger}\left(q, t_{1}\right)\right\rangle$ as the expectation value on the initial state vacuum (vacuum that is constant throughout the evolution of the system and is annihilated by $\hat{a}_{k}$ and $\hat{b}_{k}$ ) after normal ordering the $\hat{\mathbb{a}}_{k}(t)$ and $\hat{\mathbb{b}}_{k}(t)$ operators. This prescription generalizes the one for the occupation number $N_{k}=\left\langle\hat{\mathbb{b}}_{k}^{\dagger} \hat{\mathrm{b}}_{k}\right\rangle=|\beta(k, t)|^{2}$ to a generic bilinear in the field.

Using this prescription we obtain

$$
\left\langle\hat{\phi}(p, t) \hat{\phi}^{\dagger}(q, t)\right\rangle=\delta(p-q)\left[|\phi(q, t)|^{2}-\left|\phi^{\mathrm{ad}}(q, t)\right|^{2}\right] .
$$

This method therefore leads to results that are equivalent to those obtained through adiabatic regularization [22,23] and provides a physical motivation for such a procedure. Using the expression (9) of $\phi^{\text {ad }}(k, t)$ obtained to leading order in the adiabatic approximation, the renormalized result is

$$
\left\langle\hat{\phi}\left(p, t_{1}\right) \hat{\phi}^{\dagger}\left(q, t_{1}\right)\right\rangle=\delta(p-q)\left[\left|\phi\left(p, t_{1}\right)\right|^{2}-\frac{1}{2 \sqrt{\left(p+e E t_{1}\right)^{2}+m^{2}}}\right]
$$

that, substituted into (13), gives the final expression,

$$
\langle\delta E(x, t)\rangle_{(1)}=-2 e \int^{t} d t_{1} \int \frac{d p}{2 \pi}\left|\phi\left(p, t_{1}\right)\right|^{2}\left(p+e E t_{1}\right)+e \int^{t} d t_{1} \int \frac{d p}{2 \pi} \frac{p+e E t_{1}}{\sqrt{\left(p+e E t_{1}\right)^{2}+m^{2}}} .
$$

\section{Result}

The integral in Eq. (19) can be computed exactly, but we do not need to perform this calculation. It is easier to extract the physically relevant result by observing that $p$ and $t$ appear in the integrands in Eq. (19) always in the combination $(p+e E t)$, so that derivatives with respect to $t$ can be easily traded for derivatives with respect to $p: \partial / \partial t=e E \partial / \partial p$. The second derivative of Eq. (19) then reads 


$$
\begin{aligned}
\langle\delta \ddot{E}(x, t)\rangle_{(1)} & =\lim _{\Lambda_{ \pm} \rightarrow+\infty}\left[-\frac{e}{\pi} \int_{-\Lambda_{-}}^{\Lambda_{+}} d p \frac{d}{d t}\left\{|\phi(p+e E t)|^{2}(p+e E t)\right\}+\frac{e}{2 \pi} \int_{-\Lambda_{-}}^{\Lambda_{+}} d p \frac{d}{d t}\left\{\frac{p+e E t}{\sqrt{(p+e E t)^{2}+m^{2}}}\right\}\right] \\
& =-\frac{e^{2} E}{\pi} \frac{e^{-\frac{\pi m^{2}}{e E E}}}{(2 e E)^{1 / 2}} \lim _{\Lambda_{ \pm} \rightarrow+\infty}\left[\left|D_{i \frac{\pi m^{2}}{2 e E}-\frac{1}{2}}\left((p+e E t) \sqrt{\frac{2}{e E}} e^{3 i \pi / 4}\right)\right|^{2}(p+e E t)\right]_{p=-\Lambda_{-}}^{p=\Lambda_{+}}+\frac{e^{2} E}{\pi}
\end{aligned}
$$

where we have regularized the $d p$ integrals by setting the integration range on $\left(-\Lambda_{-}, \Lambda_{+}\right)$, and where we have used the fundamental theorem of integral calculus in the second line.

Using the asymptotic behavior of the parabolic cylinder function, see e.g., Eq. 9.246 of [29], we thus obtain

$$
\langle\delta \ddot{E}(x, t)\rangle_{(1)}=-\frac{e^{2} E}{\pi}\left[\left(e^{-\frac{\pi m^{2}}{e E}}+\frac{1}{2}\right)-\left(-\frac{1}{2}\right)\right]+\frac{e^{2} E}{\pi}=-\frac{e^{2} E}{\pi} e^{-\pi \frac{m^{2}}{e E}}
$$

Two comments are in order. First, if we did not subtract the adiabatic part of $\left\langle\hat{\phi}\left(p, t_{1}\right) \hat{\phi}^{\dagger}\left(q, t_{1}\right)\right\rangle$ [which results in the last term in the second line of Eq. (20)], the resulting rate (21) would not vanish in the decoupling limit $m \rightarrow \infty$. This shows the need for the subtraction of the adiabatic part of the two point function of $\phi$.

Second, the equation above gives the second time derivative of $\langle\delta E(x, t)\rangle_{(1)}$. This means that $\langle\delta E(x, t)\rangle_{(1)}$ contains two integration constants that are however unrelated to rate of pair production and thus are uninteresting for us. The first of these constants is related to the initial value of the background electric field; the second one is related to the initial value of the number of charged particles that are subsequently accelerated and decrease the background field at a rate that is linear in time. Neither of these integration constants is related to the rate of pair production that is fully captured by Eq. (21), as we now discuss.

\section{Connecting $\ddot{E}$ to the rate of pair production}

We will now connect the rate (21) of change of the electric field to the rate of production of pairs of $\phi$ particles.

If a particle of mass $m$ and charge $e$ is subject to a uniform field $E$, then its velocity is given by $v(t)=\frac{e E\left(t-t_{0}\right)}{\sqrt{m^{2}+\left(e E\left(t-t_{0}\right)\right)^{2}}}$, where $t_{0}$ is the time at which the particle is at rest.

Let now $d n_{ \pm}\left(t_{0}\right)$ be the number density of particles with charge $\pm e$ created at rest between the times $t_{0}$ and $t_{0}+d t_{0}$. Then the element of current associated to those particles and evaluated at time $t$ reads

$$
\begin{aligned}
d J\left(t, t_{0}\right)= & e d n_{+}\left(t_{0}\right) \frac{e E\left(t-t_{0}\right)}{\sqrt{m^{2}+\left(e E\left(t-t_{0}\right)\right)^{2}}} \\
& -e d n_{-}\left(t_{0}\right) \frac{(-e) E\left(t-t_{0}\right)}{\sqrt{m^{2}+\left(e E\left(t-t_{0}\right)\right)^{2}}}
\end{aligned}
$$

Using the fact that the production rates are the same for both particles and antiparticles and that they are constant in time, so that $d n_{ \pm}\left(t_{0}\right)=\dot{n} d t_{0}$ with $\dot{n}=$ constant, we get

$$
d J\left(t, t_{0}\right)=2 \dot{n} \frac{e^{2} E\left(t-t_{0}\right)}{\sqrt{m^{2}+\left(e E\left(t-t_{0}\right)\right)^{2}}} d t_{0},
$$

and finally the current at time $t$ is

$$
\begin{aligned}
J(t) & =\int_{t_{\text {in }}}^{t} \frac{d J\left(t, t_{0}\right)}{d t_{0}} d t_{0} \\
& =2 \frac{\dot{n}}{E}\left(\sqrt{m^{2}+e^{2} E^{2}\left(t-t_{\text {in }}\right)^{2}}-m\right) \rightarrow 2 \dot{n} e\left(t-t_{\text {in }}\right),
\end{aligned}
$$

where we assumed that the process started long ago, $e E\left(t-t_{\text {in }}\right) \gg m$.

Then, from the Maxwell equations $\partial_{\mu} F^{\mu \nu}=J^{\nu}$ we obtain $\dot{E}=-J \Rightarrow \ddot{E}=-\dot{J}=-2 \dot{n} e$. This finally gives

$$
\dot{n}=-\frac{\ddot{E}}{2 e}=-\frac{\delta \ddot{E}}{2 e}=\frac{e E}{2 \pi} e^{-\pi \frac{m^{2}}{e E}}
$$

in agreement with the standard result.

\section{COMPACT CASE}

Let us now consider a system where space is compactified, with the identification $x \approx x+2 \pi R$. The procedure for the compact case is similar to that presented above in the noncompact regime. As we will see, however, additional complications come from the fact we will not be able to use the fundamental theorem of integral calculus that allowed to simplify Eq. (20).

\section{A. Kaluza-Klein decomposition}

We decompose the gauge field as in the noncompact case: $\delta A(x, t)=\hat{c}(x)+\hat{d}(x) t$, with $\hat{c}(x)$ and $\hat{d}(x)$ periodic functions satisfying the commutation relations of Eq. (5). For what concern the field $\phi$, we decompose it into KaluzaKlein modes, 


$$
\begin{aligned}
\phi(t, x) & \equiv \sum_{n=-\infty}^{\infty} \frac{1}{\sqrt{2 \pi R}} e^{i n x / R} \hat{\phi}_{n}(t) \\
& =\sum_{n=-\infty}^{\infty} \frac{1}{\sqrt{2 \pi R}} e^{i n x / R}\left[\phi_{n}(t) \hat{a}_{n}+\phi_{-n}^{*}(t) \hat{b}_{-n}^{\dagger}\right]
\end{aligned}
$$

where, analogously to Eq. (10), $\phi_{n}(t)$ is given by

$$
\phi_{n}(t)=\frac{e^{-\frac{\pi m^{2}}{8 e E}}}{(2 e E)^{1 / 4}} D_{i \frac{m^{2}}{2 e E}-\frac{1}{2}}\left(-\left(\frac{n}{R}+e E t\right) \sqrt{\frac{2}{e E}} e^{-i \pi / 4}\right) .
$$

Then, substituting into Eq. (13), one obtains

$$
\begin{aligned}
\langle\delta E(x, t)\rangle_{(1)}= & -\frac{e}{\pi R} \int^{t} d t^{\prime} \sum_{n}\left|\phi_{n}\left(t^{\prime}\right)\right|^{2}\left(\frac{n}{R}+e E t^{\prime}\right) \\
& +\frac{e}{2 \pi R} \int^{t} d t^{\prime} \sum_{n} \frac{\frac{n}{R}+e E t^{\prime}}{\sqrt{\left(\frac{n}{R}+e E t^{\prime}\right)^{2}+m^{2}}} .
\end{aligned}
$$

\section{B. Result}

Since the transition to the compact case converts the integral in $d p$ in Eq. (19) into a series, we cannot use the trick-based on the fundamental theorem of calculusused in Eq. (20) to compute $\langle\delta \ddot{E}\rangle$. Instead, we have to compute the series directly. An additional complication is that the series in Eq. (28) are divergent. So we will use a different strategy. First, we take the time derivative $\langle\delta \dot{E}(x, t)\rangle_{(1)}$ that eliminates the time integral from Eq. (28).
Then we cut off the summation at some large $N_{ \pm}>0$, $-N_{-}<n<N_{+}$. We use a Mellin-Barnes representation (see Eq. 9.242.3 of [29]) of the parabolic cylinder function. This allows us to express the $n$-dependence of the first term on the right-hand side of Eq. (28) in the simple form of Hurwitz $\zeta$-like series, and the divergent part as $N_{ \pm} \rightarrow \infty$ can be isolated. The remaining part of series can be resummed to a finite result and reverse-engineered using again the Mellin-Barnes representation of the parabolic cylinder functions. The details are presented in Appendix A.

As for the vacuum part, given by the second summation on the right-hand side of Eq. (28), the analysis is simpler. Here we just state that, also in this case, we have to cut the sum off at $-N_{-}<n<N_{+}$. Then we can rewrite (Appendix B gives the details)

$$
\begin{aligned}
& \sum_{n=-N_{-}}^{N_{+}} \frac{\frac{n}{R}+e E t}{\sqrt{\left(\frac{n}{R}+e E t\right)^{2}+m^{2}}} \\
& =2 e E R t+4 m R \sum_{n=1}^{\infty} \sin (2 \pi n e E R t) K_{1}(2 \pi n m R) \\
& \quad+\text { constant, }
\end{aligned}
$$

where $K_{1}$ is the modified Bessel function of second kind, and where the constant part is generally divergent as $N_{ \pm} \rightarrow+\infty$, but does not depend on $t$.

Our final expression for the time derivative of the electric field, at first order in the perturbative expansion, reads

$$
\begin{aligned}
\langle\delta \dot{E}(t)\rangle_{(1)}= & -\frac{e}{2 \pi R}\left\{\left(1+2 e^{-\pi m^{2}}\right) \sum_{n=0}^{\infty}\left[\frac{\sqrt{2}(n+R t-[R t])}{R} e^{-\pi m^{2} / 4}\left|D_{-1 / 2+i m^{2} / 2}\left(\frac{\sqrt{2}(n+R t-[R t])}{R} e^{-i \pi / 4}\right)\right|^{2}-1\right]\right. \\
& -\sum_{n=0}^{\infty}\left[\frac{\sqrt{2}(n+1-R t+[R t])}{R} e^{-\pi m^{2} / 4}\left|D_{-1 / 2+i m^{2} / 2}\left(\frac{\sqrt{2}(n+1-R t+[R t])}{R} e^{-i \pi / 4}\right)\right|^{2}-1\right] \\
& \left.+2([R t]-R t)-4 m R \sum_{n=1}^{\infty} \sin (2 \pi n R t) K_{1}(2 \pi n m R)\right\}-\frac{e}{\pi R} e^{-\pi m^{2}}[R t]+\text { constant, }
\end{aligned}
$$

where, in order to keep the a lighter notation, we have set $e E=1$ (and we will do this in the remainder of this section and in Sec. IV), where $[x]$ denotes the integer part of $x$, and where the constant term depends on the regulators $N_{ \pm}$and is generally divergent as $N_{ \pm} \rightarrow \infty$.

Equation (30) is our main result. It gives the rate of change of electric field due to Schwinger pair production in compact spaces for arbitrary values of the parameters, at first order in the in-in expansion. In the next section we will study the behavior of this function. Here, let us note a couple of general properties. First, all the parts that are divergent as $N_{ \pm} \rightarrow+\infty$ are independent on $t$. As discussed above, these divergences correspond to a possible nonvanishing initial charge distribution in the system and are uninteresting for us. All the parts that are not explicitly dependent on $N_{ \pm}$are finite [in particular, this means that the summations appearing in Eq. (30) are convergent]. Second, the nontrivial part of Eq. (30) depends on $R t-[R t]$, so that, as a function of $t$, is periodic with a period $1 / R$. The nonperiodic component of $\langle\delta \dot{E}(t)\rangle_{(1)}$ is given by the term outside the curly brackets in Eq. (30), $-\frac{e}{\pi} e^{-\pi m^{2}} \frac{[R t]}{R}$. This means that the net rate of particle production, averaged on time scales that are long with 
respect to the time scale $R^{-1}$, coincides with the noncompact result.

\section{RESULTS FOR COMPACT CASE}

We are now in position to analyze the behavior of our main observable, $\langle\delta \dot{E}(x, t)\rangle_{(1)}$, in various regimes.

\section{A. The decompactified limit, $R \rightarrow \infty$ with fixed $m$}

Let us first make sure that our result (30) converges to the noncompact one, Eq. (21), in the limit $R \rightarrow \infty$. In this limit we note that the sums in the first two lines of Eq. (30) can be approximated by integrals, where we introduce an integration variable $p=n / R$, so that

$$
\begin{aligned}
\langle\delta \dot{E}(t)\rangle_{(1)}= & -\frac{e}{2 \pi}\left\{\left(1+2 e^{-\pi m^{2}}\right) \int_{0}^{\infty} d p\left[\sqrt{2}(p+\tau / R) e^{-\pi m^{2} / 4}\left|D_{-1 / 2+i m^{2} / 2}\left(\sqrt{2}(p+\tau / R) e^{-i \pi / 4}\right)\right|^{2}-1\right]\right. \\
& -\int_{0}^{\infty} d p\left[\sqrt{2}(p+(1-\tau) / R) e^{-\pi m^{2} / 4}\left|D_{-1 / 2+i m^{2} / 2}\left(\sqrt{2}(p+(1-\tau) / R) e^{-i \pi / 4}\right)\right|^{2}-1\right] \\
& \left.-2 \frac{\tau}{R}-4 m \sum_{n=1}^{\infty} \sin (2 \pi n R t) K_{1}(2 \pi n m R)\right\}-\frac{e}{\pi R} e^{-\pi m^{2}}[R t]+\text { constant },
\end{aligned}
$$

where we have defined the quantity,

$$
\tau \equiv R t-[R t], \quad 0 \leq \tau<1 .
$$

Next we note that $\tau / R \rightarrow 0$ in this limit, which implies that the first two lines in Eq. (31) converge to a timeindependent and irrelevant constant. Moreover, for what concerns the term involving the Bessel function $K_{1}$, one can use the asymptotic behavior $K_{1}(x) \approx \sqrt{\frac{\pi}{2 x}} e^{-x}$ to show that term vanish exponentially fast as $m R \rightarrow \infty$. As a consequence, using $\frac{[R t]}{R} \rightarrow t$ as $R \rightarrow \infty$, and reinstating, just for this result, the factors of $e E$, we reobtain the noncompact result,

$$
\langle\delta \dot{E}(t)\rangle_{(1)} \underset{R \rightarrow \infty}{\longrightarrow}-\frac{e^{2} E}{\pi} e^{-\pi m^{\frac{m^{2}}{e E}}} t+\text { constant }
$$

The result of the numerical calculation of $\langle\delta \dot{E}(1)\rangle_{(1)}$ is shown for representative values of $R$ and $m$ in Fig. 1, and agrees with the result (33).

\section{B. The Kaluza-Klein decoupling limit, $\boldsymbol{R} \rightarrow \mathbf{0}$ with fixed $\boldsymbol{m}$}

For $R \rightarrow 0$, the Kaluza-Klein modes become infinitely heavy, so that we expect each Kaluza-Klein mode of the charged fields to be separately excited. To study this regime

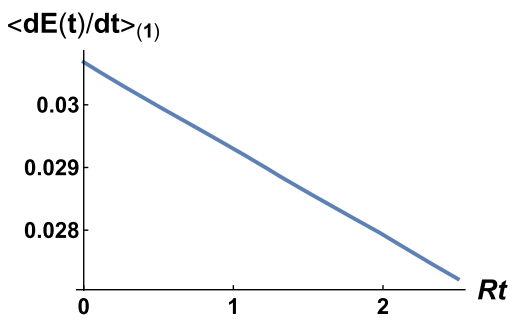

FIG. 1. The quantity $\langle\dot{E}(t)\rangle_{(1)}$, in units of $e$ and for $e E=1$, as a function of $R t$ evaluated numerically for $m=1, R=10$. The result agrees with the analytical approximation (33). analytically we assume $\frac{\tau}{R} \gg 1$ and $\frac{1-\tau}{R} \gg 1$, so that we are far enough from the instances of particle production that occur when $R t$ crosses a integer value. We can then use the asymptotic expansion of the parabolic cylinder function for large arguments, which gives

$x e^{-\pi m^{2} / 4}\left|D_{-1 / 2+i m^{2} / 2}\left(x e^{-i \pi / 4}\right)\right|^{2}-1=-\frac{m^{2}}{x^{2}}+O\left(x^{-4}\right)$.

So we get, for $R \rightarrow 0$,

$$
\begin{aligned}
\langle\delta \dot{E}(t)\rangle_{(1)} \simeq & \frac{m^{2} e R}{4 \pi}\left(1+2 e^{-\pi m^{2}}\right) \psi^{\prime}(R t-[R t]) \\
& -\frac{m^{2} e R}{4 \pi} \psi^{\prime}(1-R t+[R t]) \\
& -\frac{e}{\pi R} e^{-\pi m^{2}}[R t]+\text { constant }
\end{aligned}
$$

where $\psi^{\prime}$ denotes the derivative of the digamma function, $\psi(x)=\Gamma^{\prime}(x) / \Gamma(x), \psi^{\prime}(x)=\sum_{n=0}^{\infty}(n+x)^{-2}$, and where we have used Eq. (B7). As $R \rightarrow 0$, the terms proportional to the $\psi^{\prime}$ functions vanish, and we are left with the result,

$$
\begin{aligned}
\langle\delta \dot{E}(t)\rangle_{(1)} & \simeq-\frac{e}{\pi} e^{-\pi m^{2}} \frac{[R t]}{R}, \quad \text { with } \quad \frac{R t-[R t]}{R} \gg 1, \\
\frac{1-R t+[R t]}{R} & \gg 1, \quad R \rightarrow 0 .
\end{aligned}
$$

As stated above, this result requires $R t$ to be far from an integer value. To cover also the case where $R t$ is close to integer we have to compute $\langle\delta \dot{E}(t)\rangle_{(1)}$ numerically. We show the results (in units of $e$ ) obtained for two choices of parameters in Fig. 2. As one can see, Eq. (36) provides an excellent approximation of the exact result for $\left|\frac{R t-[R t]}{R}\right| \gg 1$, while when $R t$ is close to an integer the 

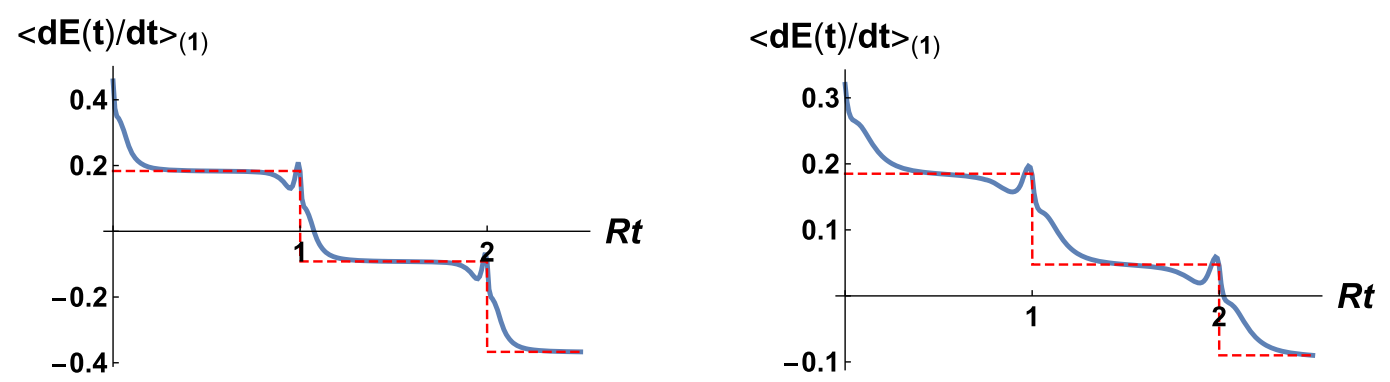

FIG. 2. The quantity $\langle\dot{E}(t)\rangle_{(1)}$, in units of $e$ and for $e E=1$, as a function of $R t$ evaluated numerically for $m=1, R=.05$ (left) and $m=1, R=.1$ (right). For $R t$ far from integer values the result agrees with the analytical approximation (36), shown in the red, dashed lines.
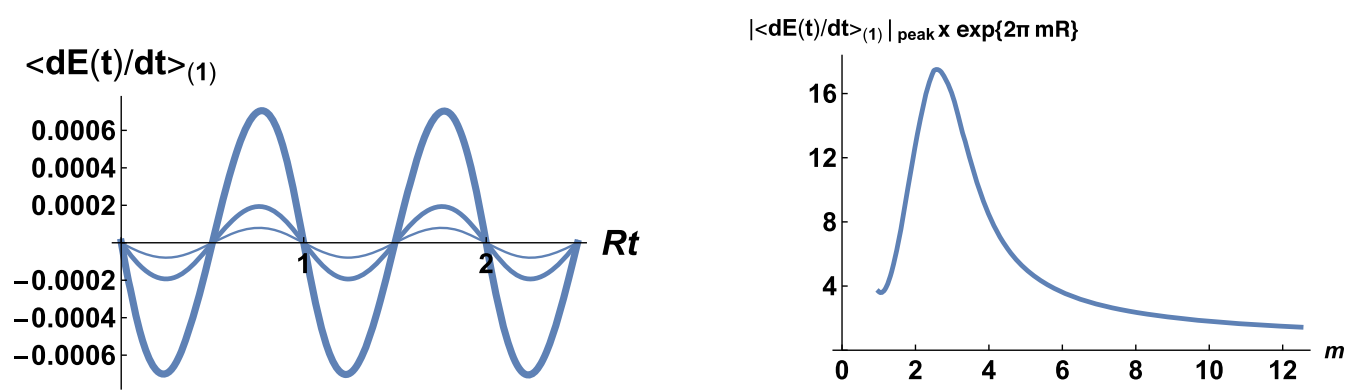

FIG. 3. Left panel: the quantity $\langle\dot{E}(t)\rangle_{(1)}$, in units of $e$ and for $e E=1$, as a function of $R t$ evaluated numerically for $m R=1$. The curves, from larger to smaller amplitude, correspond to $m=2,3,4$. Right panel: the amplitude of the oscillations of the quantity $\langle\dot{E}(t)\rangle_{(1)}$, multiplied by $e^{2 \pi m R}$, as a function of $m$ and for $R=1$.

amplitude of the correction to the electric field shows a nontrivial behavior.

\section{C. $R=\mathcal{O}(1)$ with $m R \gtrsim \mathcal{O}(1)$}

We conclude this section analyzing the case in which $m R \gtrsim \mathcal{O}(1)$, which includes the case $m R \gg 1$. We also assume $m \gtrsim 1$, which implies that the net rate of pair production, as given by the nonperiodic component of Eq. (30) is negligible. For this choice of parameters the distance in time $\sim 1 / R$ between the events of production of different Kaluza-Klein modes of the matter field is comparable to or much smaller than the duration $\sim m$ of the individual events of particle production themselves (remember that we are setting $e E=1$ in this section). To see that particle production lasts a time $\sim m$, let us remember that this process occurs when the frequency of the mode functions is evolving nonadiabatically. In our case, considering without loss of generality the zero mode of the field $\phi$, the frequency reads $\omega=\sqrt{t^{2}+m^{2}}$. Nonadiabaticity is maximal when the quantity $|\dot{\omega}| / \omega^{2}$ is maximized, which turns out to be the case when $t=m / \sqrt{2}$.

Since for $R \gtrsim \mathcal{O}(1 / \mathrm{m})$ there is always at least one Kaluza-Klein mode of the scalar field whose proper frequency is not evolving adiabatically, we find that the system is never in a fully adiabatic regime. Numerical analysis, indeed, shows a rather unusual behavior: the electric field performs sinusoidal oscillations. The amplitude of such oscillations, in the regime of large $m R \gg 1$, that can be seen to go as $e^{-2 \pi m R}$. This behavior is apparent in the plots in Fig. 3. For smaller values of $m$ and fixed $m R=\mathcal{O}(1)$ (not shown) the behavior is similar, but the oscillations are superimposed to a decay due to the term proportional to $[R t]$ at the end of Eq. (30).

In Sec. V B below we discuss the origin of this behavior.

\section{DISCUSSION AND CONCLUSIONS}

In the previous section we have analyzed the function $\langle\delta \dot{E}(t)\rangle_{(1)}$ in various regimes. We have seen that in the decompactification limit $R \rightarrow \infty$ we recover the standard result (31). In the opposite $R \rightarrow 0$ limit we have obtained a stepwise behavior, which seems inconsistent with the stationary nature of the background that would require a uniform rate of particle creation. Finally, in the case $R=\mathcal{O}(1), m R \gg 1$, the function $\langle\delta \dot{E}(t)\rangle_{(1)}$ also features an unexpected time dependence, showing oscillations whose amplitude is parametrically larger than the decrease of the electric field due to particle production.

Let us discuss the latter two results separately.

\section{A. On the steplike behavior for $\boldsymbol{R} \rightarrow \mathbf{0}$}

The steplike behavior observed in Fig. 2 is surprising if one considers that the background system displays invariance under continuous time translations. Why is this 
continuous symmetry broken down to the discrete invariance under $t \mapsto t+1 /(e E R)$, and what does determine the exact time when particle production occurs?

The fact that vacuum decay might break some of the background spacetime symmetries has been already discussed, for instance in [7,30,31]. Analogously to what was shown in [7], which focused on the Lorentz invariance of the Schwinger phenomenon in noncompact space, the vacuum of our system is invariant under time translation, as a time translation $t \mapsto t+\delta t$ is canceled by a gauge transformation $A \mapsto A-E \delta t$. Conversely, the addition of a constant to the gauge potential would determine a shift in the times of particle production. In compact space, a physical observable can have gauge dependence, as in the Aharonov-Bohm effect, which implies that gauge transformations can also be observable unless they meet certain conditions. In our case, gauge invariance is preserved if the particle picks up a phase equal to a multiple of $2 \pi$, which corresponds to a time translation $\delta t$ is equal to a multiple of $\frac{1}{e E R}$, and which in its turn corresponds to a shift of the Kaluza-Klein modes by an integer. Choosing a gauge, as we did, is equivalent to choosing an initial time for a particular observer who measures the particle productions, analogously to the choice of an observer's frame for bubble nucleation, as discussed in [7]. Finally, it is worth noting that an exactly time-translational invariant electric field is an idealization-if this state is decaying then it cannot have been around forever, otherwise it would have already completely decayed. An electric field must be turned on at some initial time, which will break the time translation symmetry manifestly.

\section{B. On the oscillations for $e E R^{2}=\mathcal{O}(1), m^{2} \gg e E$}

The sinusoidal time dependence observed in the regime $e E R^{2}=\mathcal{O}(1), m^{2} \gg e E$ can be explained by invoking the $(0+1)$-dimensional effective description of the model, as done in [11]. In fact, in this regime particle production is negligible, and one can just study the theory that results by integrating out the heavy Kaluza-Klein modes of the bulk charged matter. Such an operation leads to a $(0+1)$ dimensional Lagrangian that in the limit $m R \gg 1$ reads

$L_{0+1, \mathrm{eff}} \simeq \pi R \dot{A}_{0}(t)^{2}+\frac{m^{1 / 2}}{\pi R^{1 / 2}} e^{-2 \pi m R} \cos \left(2 \pi R e A_{0}(t)\right)$,

where $A_{0}(t) \equiv \int_{0}^{2 \pi R} \frac{d x}{2 \pi R} A(t, x)$. For a derivation of Eq. (37) see e.g., [11], that works with a $(4+1)$-dimensional theory. To obtain the effective potential derived from our $(1+1)$ dimensional theory one should multiply the effective potential in that paper by $(2 \pi L / m)^{3 / 2}$ with $L=2 \pi R$ to match our notation. Then, by solving the classical equations of motion derived from the Lagrangian (37) to first order in the small quantity $e^{-2 \pi m R}$ and identifying $E(t)=-\dot{A}_{0}$ we obtain

$$
\begin{aligned}
E(t)= & E+e \frac{m^{1 / 2}}{2 \pi^{2} R^{3 / 2} e E} e^{-2 \pi R m} \cos (2 \pi R e E t) \\
& +\mathcal{O}\left(e^{-4 \pi R m}\right),
\end{aligned}
$$

which displays oscillations that, remarkably, have the same periodicity and whose amplitude has the same overall proportionality to $e^{-2 \pi m R}$ as the result found in Sec. IV C. Actually, the oscillations in Eq. (38) match exactly, including $\mathcal{O}(1)$ factors, the result from the adiabatic component of $\langle\delta \dot{E}(t)\rangle_{(1)}$, presented in Eq. (B8). This is not too surprising, as the effective potential in Eq. (37) has been obtained using the semiclassical approximation. Going beyond the overall proportionality to $e^{-2 \pi m R}$, however, the behavior found in the analysis of Sec. IV C above shows a different dependence on $m$ and $R$ than that of Eq. (B8). This can be seen, for instance, from the fact the amplitude shown in the right panel of Fig. 3 is a decreasing function of $m$, whereas the result (B8) increases as $m^{1 / 2}$, once the overall proportionality to $e^{-2 \pi m R}$ is factored out. This means that both the adiabatic contribution evaluated in Appendix B and the full one, obtained in Appendix A, have the same overall sinusoidal behavior $\propto \cos (2 \pi e E R t)$, but a different amplitude. Inspection of those two terms in the $R=\mathcal{O}(1), m \gg 1$ regime shows that the they partially cancel, with the full component larger than the adiabatic one. It is possible that this difference is an artifact of the finiteness of the mass $m$ in our numerical calculations and that the result will match exactly Eq. (38) in the limit $m \rightarrow \infty$.

Our analysis thus confirms, via a real time calculation performed in the full $(1+1)$-dimensional theory, the result presented in [11], which in its turn explains, in terms of the compactified theory, the observation of [10] that, for small compactification radii, a new instanton with magnitude $\sim e^{-2 \pi m R}$ would dominate the Schwinger effect. In particular, our analysis shows that the net rate of pair production is proportional to $e^{-\pi \frac{m^{2}}{e E}}$ also for small compactification ${ }^{1}$ radii, but for short time scales the effect associated to particle creation is subdominant with respect to the oscillations, with amplitude proportional to $e^{-2 \pi m R}$ and frequency $R / e E$, induced by the virtual charged matter.

To conclude, we have found real time formulas that allow us to compute the change in the electric field due to

\footnotetext{
${ }^{1}$ It is worth stressing that our analysis has been performed to leading order in the in-in expansion, leading to a result $\langle\delta E(t)\rangle_{(1)}=e f_{(1)}(e E, m, R, t)$. According to [10], the new instanton should be associated to a final state with a lower electric field, but no matter particles. In our in-in language, this would correspond to a higher order term $\langle\delta E(t)\rangle_{(3)}=e^{3} f_{(3)}(e E, m, R, t)$ in the expansion (11) that accounts for $\phi$ annihilation. It is thus not excluded that a net contribution to the rate of pair production proportional to $e^{-2 \pi m R}$ might appear at that order in perturbation theory. See [19] for the discussion of an analogous phenomenon in the thermal case, and [8,9] for a higher order real-time calculation for the zero-temperature, noncompact case.
} 
Schwinger effect in a compact space. The net rate of production of charged pairs, when evaluated on long times, is always proportional to $e^{-\pi \frac{m^{2}}{e E}}$, as in the non compact case. However, while in the limit of large compactification radii we recover the expected noncompact result; in the regime of intermediate and small values of the dimensionless quantity $m R$ the quantity $\langle\delta E(t)\rangle_{(1)}$ shows a richer behavior. In particular, the steplike time dependence found in the regime of $m R \rightarrow 0$ breaks continuous time translations in a fashion that is analogous to the way bubble nucleation breaks Lorentz symmetry, and the oscillations found for $m^{2} \gg e E, R^{2}=\mathcal{O}\left((e E)^{-1}\right)$ can be explained as an effect of the virtual pairs of charged particles on the effective potential for the gauge field.

\section{ACKNOWLEDGMENTS}

We thank Patrick Draper, Gerald Dunne, Oliver Gould, David Kastor, Matt Kleban, and Jennie Traschen for interesting discussions. This work is partially supported by the US-NSF Grants No. PHY-1520292 and No. PHY1820675.

\section{APPENDIX A: CALCULATION FOR COMPACT CASE}

In this Appendix we work out the steps that allow us to go from the first term on the right-hand side of Eq. (28) to the right-hand side of Eq. (30) (except for the part proportional to the Bessel function $K_{1}$, that will be discussed in Appendix B below).
We set $e E=1$, and focus on the sum,

$$
\begin{aligned}
\mathcal{S}= & \frac{1}{R} \sum_{n=-\infty}^{\infty} \frac{e^{-\pi m^{2} / 4}}{\sqrt{2}}\left|D_{i \frac{m^{2}}{2}-\frac{1}{2}}\left(-(n / R+t) \sqrt{2} e^{-i \pi / 4}\right)\right|^{2} \\
& \times(n / R+t),
\end{aligned}
$$

so that

$$
\langle\delta \dot{E}(t)\rangle_{(1)}=-\frac{e}{\pi} \mathcal{S}+\frac{e}{2 \pi R} \sum_{n=-\infty}^{\infty} \frac{n / R+e E t}{\sqrt{(n / R+e E t)^{2}+m^{2}}} .
$$

We want to use the Mellin-Barnes representation, Eq. 9.242.3 of [29], of the parabolic cylinder function,

$$
D_{\nu}(z)=\frac{e^{-\frac{1}{4} z^{2}} z^{\nu}}{2 \pi i \Gamma(-\nu)} \int_{-i \infty}^{i \infty} \Gamma(t) \Gamma(-\nu-2 t) 2^{t} z^{2 t} d t,
$$

that is valid for $|\operatorname{Arg}(z)|<3 \pi / 4$. Given this restriction on $\operatorname{Arg}(z)$, we have to treat the cases $(n / R+t)>0$ and $(n / R+t)<0$ separately, so that we write $\mathcal{S} \equiv \mathcal{S}_{-}+\mathcal{S}_{+}$ where $n$ in $\mathcal{S}_{-}$goes from $-N_{-}$to $[-R t]-1$ (we define here the integer part in such a way that $[-x]=-[x])$, and in $\mathcal{S}_{+}$ goes from $[-R t]$ to $N_{+}$. Here, $N_{ \pm}>0$ are regulators that we will eventually send to infinity.

Let us first consider $\mathcal{S}_{-}$, where $-(n / R+t) \sqrt{2} \equiv$ $|z|>0$. In this case we can use Eq. (A3) right away. It is convenient to write the integral (A3) as an asymptotic series on the poles at $t=-j, j=0,1,2, \ldots$

$$
D_{i a-1 / 2}\left(|z| e^{-i \pi / 4}\right)=\frac{e^{\frac{i}{\mid}|z|^{2}}|z|^{i a-\frac{1}{2}} e^{\pi a / 4+i \pi / 8}}{\Gamma\left(\frac{1}{2}-i a\right)} \sum_{j=0}^{\infty} \frac{(-1)^{j}}{j !} \Gamma\left(\frac{1}{2}-i a+2 j\right) 2^{-j}|z|^{-2 j} e^{i \pi j / 2},
$$

that allows us to write, after relabeling $n \rightarrow-n$,

$$
\mathcal{S}_{-}=-\frac{\cosh \left(\pi m^{2} / 2\right)}{2 \pi R} \sum_{n^{\prime}=[R t]+1}^{N_{-}} \sum_{j, k=0}^{\infty} \frac{(-1)^{j}}{j !} \frac{(-1)^{k}}{k !} \Gamma\left(\frac{1}{2}-i \frac{m^{2}}{2}+2 j\right) \Gamma\left(\frac{1}{2}+i \frac{m^{2}}{2}+2 k\right)\left(\frac{R}{2}\right)^{2 j+2 k} \frac{e^{i \pi(j-k) / 2}}{\left(n^{\prime}-R t\right)^{2 j+2 k}} .
$$

Then, we separate the sum into the components with $j=k=0, j=0$ and $k \geq 1, j \geq 1$ and $k=0$, and $j, k \geq 1$,

$$
\begin{aligned}
\mathcal{S}_{-}= & -\left\{\frac{1}{2 R} \sum_{n^{\prime}=[R t]+1}^{N_{-}} 1\right\}-2 \Re\left\{\frac{1}{2 R} \sum_{n^{\prime}=[R t]+1}^{N_{-}} \sum_{k=1}^{\infty} \frac{(-1)^{k}}{k !} \frac{\Gamma\left(\frac{1}{2}+i \frac{m^{2}}{2}+2 k\right)}{\Gamma\left(\frac{1}{2}+i \frac{m^{2}}{2}\right)}\left(\frac{R}{2}\right)^{2 k} \frac{1}{\left(n^{\prime}-R t\right)^{2 k}} e^{i \pi(-k) / 2}\right\} \\
& -\frac{1}{2 R} \sum_{n^{\prime}=[R t]+1}^{N_{-}} \sum_{j, k=1}^{\infty} \frac{(-1)^{j}}{j !} \frac{(-1)^{k}}{k !} \frac{\Gamma\left(\frac{1}{2}+i \frac{m^{2}}{2}+2 k\right)}{\Gamma\left(\frac{1}{2}+i \frac{m^{2}}{2}\right)} \frac{\Gamma\left(\frac{1}{2}-i \frac{m^{2}}{2}+2 j\right)}{\Gamma\left(\frac{1}{2}+i \frac{m^{2}}{2}\right)}\left(\frac{R}{2}\right)^{2 j+2 k} \frac{e^{i \pi(j-k) / 2}}{\left(n^{\prime}-R t\right)^{2 j+2 k}} .
\end{aligned}
$$

This allows us to isolate the divergence in the limit $N_{-} \rightarrow \infty$, that appears only in the first term of the equation above. We can now send $N_{-} \rightarrow \infty$ in the remaining terms, shift the summation variable $n$ by $[R t]+1$, and write

$$
\mathcal{S}_{-}=-\frac{N_{-}-[R t]-1}{2 R}-\frac{1}{2 R} \sum_{n=0}^{\infty}\left[|\mathcal{G}+1|^{2}-1\right]
$$


where

$$
\mathcal{G}=\sum_{j=1}^{\infty} \frac{(-1)^{j}}{j !} \frac{\Gamma\left(\frac{1}{2}-i \frac{m^{2}}{2}+2 j\right)}{\Gamma\left(\frac{1}{2}-i \frac{m^{2}}{2}\right)}\left(\frac{1}{2}\right)^{j} \frac{1}{[\sqrt{2}(n+[R t]+1-R t) / R]^{2 j}} e^{i \pi j / 2},
$$

that, using again Eq. (A4), gives

$$
|\mathcal{G}+1|^{2}=\sqrt{2} \frac{n+[R t]+1-R t}{R} e^{-\pi m^{2} / 4}\left|D_{i m^{2} / 2-1 / 2}\left(\sqrt{2} \frac{n+[R t]+1-R t}{R} e^{-i \pi / 4}\right)\right|^{2} .
$$

Next, we have to take care of $\mathcal{S}_{+}$, where the phase of the argument of the parabolic cylinder function is precisely $3 \pi / 4$, so that the expression (A3) is not directly applicable. In order to use Eq. (A3) we have to first apply the third of Eqs. 9.248.1 of [29],

$$
D_{p}(z)=e^{i \pi p} D_{p}(-z)+\frac{\sqrt{2 \pi}}{\Gamma(-p)} e^{i(p+1) \pi / 2} D_{-p-1}(-i z),
$$

so that $\mathcal{S}_{+}$reads

$$
\begin{aligned}
\mathcal{S}_{+}= & \frac{1}{2 R} \sum_{n=[-R t]}^{N_{+}}\left[e^{-5 \pi m^{2} / 4}\left|D_{i \frac{m^{2}}{2}-\frac{1}{2}}\left(|n / R+t| \sqrt{2} e^{-i \pi / 4}\right)\right|^{2} \sqrt{2}(n / R+t)\right. \\
& +e^{-3 \pi m^{2} / 4}\left|\frac{\sqrt{2 \pi}}{\Gamma\left(-i \frac{m^{2}}{2}+\frac{1}{2}\right)} D_{-i \frac{m^{2}}{2}-\frac{1}{2}}\left(|n / R+t| \sqrt{2} e^{i \pi / 4}\right)\right|^{2} \sqrt{2}(n / R+t) \\
& \left.+2 \sqrt{2} e^{-\pi m^{2}} \mathfrak{R}\left\{-i D_{i \frac{m^{2}}{2}-\frac{1}{2}}\left(|n / R+t| \sqrt{2} e^{-i \pi / 4}\right) \frac{\sqrt{2 \pi} e^{-i \pi / 4}}{\Gamma\left(i \frac{m^{2}}{2}+\frac{1}{2}\right)} D_{i \frac{m^{2}}{2}-\frac{1}{2}}\left(|n / R+t| \sqrt{2} e^{-i \pi / 4}\right)(n / R+t)\right\}\right] .
\end{aligned}
$$

The summand in the third line of this expression is quickly oscillating, and we neglect it. The terms in the first two lines can be treated in a way that is analogous to the one that led to Eqs. (A7) and (A9).

We thus obtain the desired result,

$$
\begin{aligned}
\mathcal{S}= & \frac{1}{2 R}\left\{\left(1+2 e^{-\pi m^{2}}\right) \sum_{n=0}^{\infty}\left[\frac{\sqrt{2}(n+R t-[R t])}{R} e^{-\pi m^{2} / 4}\left|D_{-1 / 2+i m^{2} / 2}\left(\frac{\sqrt{2}(n+R t-[R t])}{R} e^{-i \pi / 4}\right)\right|^{2}-1\right]\right. \\
& -\sum_{n=0}^{\infty}\left[\frac{\sqrt{2}(n+1-R t+[R t])}{R} e^{-\pi m^{2} / 4}\left|D_{-1 / 2+i m^{2} / 2}\left(\frac{\sqrt{2}(n+1-R t+[R t])}{R} e^{-i \pi / 4}\right)\right|^{2}-1\right] \\
& \left.+\left(2+2 e^{-\pi m^{2}}\right)[R t]+\left(1+2 e^{-\pi m^{2}}\right)\left(1+N_{+}\right)-N_{-}\right\},
\end{aligned}
$$

where the sums over $n$ are finite, and the divergences in the terms containing $N_{ \pm}$have been isolated.

\section{APPENDIX B: THE VACUUM CONTRIBUTION}

The contribution to $\langle\delta \dot{E}(x, t)\rangle_{(1)}$ from the vacuum can be read from Eq. (28) and takes the form,

$$
\frac{e}{2 \pi R} \sum_{n=-N_{-}}^{N_{+}} \frac{\frac{n}{R}+e E t}{\sqrt{\left(\frac{n}{R}+e E t\right)^{2}+m^{2}}}
$$

This sum is divergent as $N_{ \pm} \rightarrow+\infty$, but its derivative with respect to $R t$ is convergent, so that we can take the limit $N_{ \pm} \rightarrow+\infty$ after differentiation and obtain (after setting $e E=1$ ) 


$$
\begin{aligned}
& \frac{e}{2 \pi R} \frac{\partial}{\partial(R t)}\left\{\sum_{n=-N_{-}}^{N_{+}} \frac{n+R t}{\sqrt{(n+R t)^{2}+(\mathrm{Rm})^{2}}}\right\}=\frac{e}{2 \pi R} \sum_{n=-\infty}^{\infty} \frac{(m R)^{2}}{\left[(n+R t)^{2}+(m R)^{2}\right]^{3 / 2}} \\
& \quad=\frac{e}{2 \pi R} \frac{(m R)^{2}}{\Gamma(3 / 2)} \int_{0}^{\infty} d w w^{1 / 2} \sum_{n=-\infty}^{\infty} e^{-w\left[(n+R t)^{2}+(m R)^{2}\right]}=\frac{e}{\pi R}(m R)^{2} \int_{0}^{\infty} d w e^{-w(m R)^{2}} \theta_{3}\left(\pi R t, e^{-\pi^{2} / w}\right),
\end{aligned}
$$

where $\theta_{3}$ denotes the third Jacobi $\theta$ function. Using the representation $\theta_{3}(u, q)=1+2 \sum_{n=1}^{\infty} q^{n^{2}} \cos (2 n u)$, we can write

$$
\frac{e}{2 \pi R} \frac{\partial}{\partial(R t)}\left\{\sum_{n=-N_{-}}^{N_{+}} \frac{n+R t}{\sqrt{(n+R t)^{2}+(\mathrm{Rm})^{2}}}\right\}=\frac{e}{2 \pi R}\left[2+4(m R)^{2} \sum_{n=1}^{\infty} \cos (2 \pi n R t) \frac{2 \pi n}{m R} K_{1}(2 \pi n m R)\right],
$$

that is a more transparent sum. Integrating back in $d(R t)$, we obtain

$$
\frac{e}{2 \pi R} \sum_{n=-N_{-}}^{N_{+}} \frac{n+R t}{\sqrt{(n+R t)^{2}+(\mathrm{Rm})^{2}}}=\frac{e}{\pi R}\left[R t+2 m R \sum_{n=1}^{\infty} \sin (2 \pi n t R) K_{1}(2 \pi n m R)+\text { constant }\right],
$$

where the constant is generally divergent, as it depends on the cutoffs $N_{ \pm}$.

In the limit $m R \rightarrow 0$ we can use the small argument approximation of the Bessel function, $K_{1}(x) \simeq 1 / x$, to write

$$
\begin{aligned}
\left.\frac{e}{2 \pi R} \sum_{n=-N_{-}}^{N_{+}} \frac{n+R t}{\sqrt{(n+R t)^{2}+(\mathrm{Rm})^{2}}}\right|_{m R \ll 1} & \simeq \frac{e}{\pi R}\left[R t+\sum_{n=1}^{\infty} \frac{\sin (2 \pi n t R)}{n \pi}+\text { constant }\right] \\
& =\frac{e}{\pi R}\left[R t+\frac{1-2 t R}{2}+\text { constant }\right]=\text { constant }
\end{aligned}
$$

where the approximation is valid for $t R \gg m R$. Of course, for $t R=0$ and $t R=1, \sin (2 \pi n t R)=0$, so

$$
\left.\frac{e}{2 \pi R} \sum_{n=-N_{-}}^{N_{+}} \frac{n+R t}{\sqrt{(n+R t)^{2}+(\mathrm{Rm})^{2}}}\right|_{t R=1}-\left.\frac{e}{2 \pi R} \sum_{n=-N_{-}}^{N_{+}} \frac{n+R t}{\sqrt{(n+R t)^{2}+(\mathrm{Rm})^{2}}}\right|_{t R=0}=\frac{e}{\pi R},
$$

which implies

$$
\left.\frac{e}{2 \pi R} \sum_{n=-N_{-}}^{N_{+}} \frac{n+R t}{\sqrt{(n+R t)^{2}+(\mathrm{Rm})^{2}}}\right|_{m R \ll 1} \simeq \frac{e}{\pi R}[t R]+\text { constant }
$$

On the other hand, in the large $2 \pi m R$ limit, we use the asymptotics $K_{1}(x) \simeq \sqrt{\frac{\pi}{2 x}} e^{-x}$ to keep only the first term in the series, obtaining

$$
\left.\frac{e}{2 \pi R} \sum_{n=-N_{-}}^{N_{+}} \frac{n+R t}{\sqrt{(n+R t)^{2}+(\mathrm{Rm})^{2}}}\right|_{m R \gg 1}=\frac{e}{\pi R}\left[R t+\sqrt{m R} \sin (2 \pi t R) e^{-2 \pi m R}+\text { constant }\right] .
$$


[1] F. Sauter, Z. Phys. 69, 742 (1931).

[2] J. S. Schwinger, Phys. Rev. 82, 664 (1951).

[3] J. D. Brown and C. Teitelboim, Phys. Lett. B 195, 177 (1987).

[4] A. I. Nikishov, Zh. Eksp. Teor. Fiz. 57, 1210 (1969) [Sov. Phys. JETP 30, 660 (1970)].

[5] Y. Kluger, E. Mottola, and J. M. Eisenberg, Phys. Rev. D 58, 125015 (1998).

[6] N. Tanji, Ann. Phys. (Amsterdam) 324, 1691 (2009).

[7] J. Garriga, S. Kanno, M. Sasaki, J. Soda, and A. Vilenkin, J. Cosmol. Astropart. Phys. 12 (2012) 006.

[8] E. Akhmedov, N. Astrakhantsev, and F. Popov, J. High Energy Phys. 09 (2014) 071.

[9] E. Akhmedov and F. Popov, J. High Energy Phys. 09 (2015) 085 .

[10] A. R. Brown, Phys. Rev. D 98, 036008 (2018).

[11] P. Draper, Phys. Rev. D 98, 125014 (2018).

[12] C. Nagele, J. E. Cejudo, T. Byrnes, and M. Kleban, Phys. Rev. D 99, 094501 (2019).

[13] D. Karabali, S. Kürkcüoğlu, and V. Nair, Phys. Rev. D 100, 065005 (2019).

[14] D. Karabali, S. Kürkcüoğlu, and V. Nair, Phys. Rev. D 100, 065006 (2019).

[15] L. Medina and M. C. Ogilvie, Phys. Rev. D 95, 056006 (2017).

[16] O. Gould and A. Rajantie, Phys. Rev. D 96, 076002 (2017).

[17] O. Gould, A. Rajantie, and C. Xie, Phys. Rev. D 98, 056022 (2018).
[18] M. Korwar and A. M. Thalapillil, Phys. Rev. D 98, 076016 (2018).

[19] G. Torgrimsson, Phys. Rev. D 99, 096007 (2019).

[20] S. Weinberg, Phys. Rev. D 72, 043514 (2005).

[21] J. M. Maldacena, J. High Energy Phys. 05 (2003) 013.

[22] L.E. Parker and D. Toms, Quantum Field Theory in Curved Spacetime: Quantized Field and Gravity (Cambridge University Press, Cambridge, England, 2011).

[23] N. D. Birrell and P.C.W. Davies, Quantum Fields in Curved Space (Cambridge University Press, Cambridge, England, 1982).

[24] M. V. Berry, Proc. R. Soc. Lond. A 422, 7 (1989).

[25] A. I. Nikishov and V. I. Ritus, Theor. Math. Phys. 92, 711 (1992).

[26] C. K. Dumlu and G. V. Dunne, Phys. Rev. Lett. 104, 250402 (2010).

[27] R. Dabrowski and G. V. Dunne, Phys. Rev. D 90, 025021 (2014).

[28] R. Dabrowski and G. V. Dunne, Phys. Rev. D 94, 065005 (2016).

[29] I. S. Gradshteyn and I. M. Ryzhik, Table of Integrals, Series, and Products, 7th ed. (Elsevier/Academic Press, Amsterdam, 2007).

[30] J. Garriga, B. Shlaer, and A. Vilenkin, J. Cosmol. Astropart. Phys. 11 (2011) 035.

[31] M. Dine, P. Draper, and C. Park, Phys. Rev. D 86, 065033 (2012). 